\title{
Revisit the calibration errors on experimental slant total electron content (TEC) determined with GPS
}

\author{
Wenfeng $\mathrm{Nie}^{1,2,3}$, Tianhe $\mathrm{Xu}^{1,2^{*}}$, Adria Rovira-Garcia ${ }^{4 *}$, José Miguel Juan Zornoza ${ }^{4}$, \\ Jaume Sanz Subirana ${ }^{4}$, Guillermo González Casado ${ }^{4}, \mathrm{Wu} \mathrm{Chen}^{3}$, Guochang $\mathrm{Xu}^{1}$ \\ ${ }^{1}$ Institute of Space Sciences, Shandong University, Weihai, China \\ ${ }^{2}$ State Key Laboratory of Geo-information Engineering, Xi'an, China \\ ${ }^{3}$ Department of Land Surveying and Geo-Informatics, The Hong Kong
}

Polytechnic University, Hong Kong, China

${ }^{4}$ Research Group of Astronomy and Geomatics (gAGE), Universitat

Politecnica de Catalunya (UPC), Barcelona, Spain

*Corresponding author at Institute of Space Sciences, Shandong University;

Research Group of Astronomy and Geomatics (gAGE), Universitat Politecnica de

Catalunya (UPC)

Email: thxugie@163.com; adria.rovira@upc.edu

ORCID code:

Wenfeng Nie: 0000-0002-5505-2903

Tianhe Xu: 0000-0001-5818-6264

Adria Rovira-Garcia: 0000-0002-7320-5029

José Miguel Juan Zornoza: 0000-0003-1126-2367

Jaume Sanz Subirana: 0000-0001-8880-7084

Guillermo Gonzalez Casado: 0000-0001-6765-2407

Wu Chen: 0000-0002-1787-5191

Guochang Xu: 0000-0002-1509-4666 
Abstract:

The calibration errors on experimental Slant Total Electron Content (STEC) determined with Global Positioning System (GPS) observations is revisited. Instead of the analysis of the calibration errors on the carrier phase leveled to code ionospheric observable, we focus on the accuracy analysis of the undifferenced ambiguity-fixed carrier phase ionospheric observable determined from a global distribution of permanent receivers. The results achieved are: (1) By using data from an entire month within the last Solar Cycle maximum, the undifferenced ambiguity-fixed carrier phase ionospheric observable is found to be over one order of magnitude more accurate than the carrier phase leveled to code ionospheric observable and the raw code ionospheric observable. The observation error of the undifferenced ambiguity-fixed carrier phase ionospheric observable ranges from 0.05 to 0.11 TECU (Total Electron Content Unit) while that of the carrier phase leveled to code and the raw code ionospheric observable is from 0.65 to 1.65 TECU and 3.14 to 7.48 TECU, respectively. (2) The time-varying receiver DCB, which presents clear Day Boundary Discontinuity and intra-day variability pattern, contributes the most part of the observation error. This contribution is assessed by the short-term stability of the Between-Receiver DCB, which ranges from 0.06 to 0.17 TECU in a single day. (3) The remaining part of the observation errors presents a sidereal time cycle pattern, indicating the effects of the multipath. Further, the magnitude of the remaining part implies that the code multipath effects are much reduced. (4) The intra-day variation of the Between-Receiver DCB of the collocated stations suggests that estimating DCBs as a daily constant can have a mis-modeling error of at least several tenths of one TECU.

Key words: ionospheric observable, total electron content, integer ambiguity resolution, receiver DCB 


\section{Introduction}

The Total Electron Content (TEC) of the ionosphere, corresponding to the atmospheric layer between 60 and $2000 \mathrm{~km}$ in height, can be studied with a linear combination of measurements from Global Navigation Satellite Systems (GNSSs). This combination is known as ionospheric observable because it cancels the non-frequency dependent terms: the geometric distance between the receiver and the satellite, the satellite and receiver clocks, and the tropospheric delay. Thus, the remaining terms are frequency-dependent: the Slant TEC (STEC) and Differential Code Bias (DCB) of the receiver and of the satellite.

Two types of measurements are commonly used to derive the ionospheric observable. On the one hand, the pseudorange measurements that are based on the code delay. This raw pseudorange measurement, termed as RP4 hereafter, contains the sum of STEC and DCB, but it is affected by noise up to several TECUs. For the Global Positioning System (GPS) L1 observable, 1 TECU (Total Electron Content Unit) corresponds to $0.162 \mathrm{~m}$. On the other hand, the carrier phase measurements are two orders of magnitude more precise than the code (Bossler et al. 1980; Braasch 1996). However, the carrier phase measurements contain an unknown term, which is the so-called ambiguity, composed by an integer number of wavelengths. The value of the ambiguity changes every time that the receiver locks the GNSS signal of the satellite. Fortunately, it remains constant until the next tracking discontinuity occurs.

Several techniques are used to estimate the ambiguity, and then use the carrier phase measurements as precise unambiguous pseudoranges to retrieve the sum of STEC and DCB. The most widely used method is the carrier phase to code leveling process (Wilson and Manucci 1993; Manucci et al. 1999). The ambiguity term is estimated by averaging the difference between the carrier phase and the code per each continuous arc. The accuracy of the carrier phase leveled to code ionospheric observable, which is also called smoothed pseudorange geometry-free combination, "SP4" for short, ranging from 1.4 to 5.3 TECU, is mainly affected by leveling errors introduced from the code multipath effect (Ciraolo et al. 2007; Brunini and 
Azpilicueta, 2009). The simplicity and effectivity of the leveling process make it preferred by most of the Ionosphere Associate Analysis Centers (IAACs) belonging to the International GNSS Service (IGS) (Dow et al. 2009; Hernández-Pajares et al. 2016).

As the demand for high accuracy ionospheric observable increases, the ionospheric observable retrieval based on Precise Point Positioning (PPP) technique (Zumberge et al. 1997) has gained interests of the ionospheric research community. Zhang et al. (2012) and Zhang (2016) reported extracting the ionospheric observable using the undifferenced and uncombined PPP technique. Further, Liu et al. (2017) extended the retrieval of the ionospheric observable from GPS-only PPP to Multi-GNSS PPP using the raw observations. Moreover, Liu et al. (2018) implemented the ionospheric observable retrieval from the post-processing to the real-time mode. The aforementioned results are based on the float ambiguity resolution of PPP. With the development of PPP Integer Ambiguity Resolution (IAR) (Laurichesse and Mercier 2007; Collins et al. 2008; Ge et al. 2008), Banville et al. (2012) used the integer-leveled observation based on the decoupled clocks (Collins et al. 2008) to monitor the ionosphere, while Rovira-Garcia et al. (2015, 2016a) extracted the ionospheric observable based on the Fast-Precise Point Positioning technique (Fast-PPP), in which the undifferenced fixed ambiguities are determined from a global distribution of permanent receivers (Juan et al. 2012). In these works, the fixed undifferenced ambiguity terms are substituted into the geometry-free combination of carrier phases, which is usually called L4 observable, and the sum of STEC and DCB can then be retrieved from the undifferenced ambiguity-fixed carrier phase ionospheric observable, termed FL4 observable hereafter.

Regardless of the methods used to estimate the ambiguity, the STEC needs to be separated from satellite and receiver DCBs. Typical values of -11 to 14 TECU, that is -4 to $5 \mathrm{~ns}$ with $1 \mathrm{~ns}$ corresponding to 2.86 TECU on GPS L4 observable, have been found for the DCB of the satellites, while the receiver DCBs range from -57 to 43 TECU (Hernández-Pajares et al. 2009). It is generally accepted that the satellite DCB 
111 is more stable than the receiver DCB (Sardon et al. 1994; Sardon and Zarraoa 1997).

112 Taking the year 2014 as an example, satellite DCBs provided by IAAC exhibited a

113 standard deviation of 0.34 to 0.57 TECU, whereas receiver DCB stabilities at the level

114 of 0.86 to 1.43 TECU were reported (Sanz et al. 2017). Also, variations in the receiver

115 DCB ranging from 1.4 to 8.8 TECU in intervals of hours have been reported (Ciraolo

116 et al. 2007). This variation is thought to be attributed to temperature changes of the

117 receiver hardware, the chain composed by the hardware in the pre-amplifier of the

118 antenna and the cable connecting to the antenna (Coster et al. 2013; Zhang and

119 Teunissen, 2015; Zhang et al. 2017).

120 We quantify the observation errors of the RP4, SP4 and FL4 ionospheric 121 observables and then focus on the contribution of the time-varying receiver DCB to 122 the observation errors. First, the procedure to obtain the different ionospheric 123 observables is summarized, and then the method to assess the accuracy of the 124 ionospheric observable throughout an experiment with collocated stations is described. 125 After the introduction of the experimental data, the results and discussions of the 126 accuracy assessment of the ionospheric observable, as well as the contribution of the 127 receiver DCB are presented. Finally, we summarize the results and gather the 128 conclusions.

\section{The ionospheric observables}

This section reviews the different procedures to retrieve the aforementioned ionospheric observables that have been used in the present study, in ascending level of accuracy: RP4, SP4 and FL4. The equations used in the derivation of the observables are explained in more detail than in Nie et al. (2018), emphasizing the description of the strategies employed in the resolution of the carrier phase ambiguity term.

137 Code and carrier phase ionospheric observable (P4 and L4) 
noted that the ionospheric range delay for code ionospheric observations $P_{4}$ has opposite sign than for carrier phase ionospheric observable $L_{4}$,

$$
\begin{gathered}
P_{4}=P_{2}-P_{1}=I+K_{21}+\varepsilon_{P 4} \\
L_{4}=\Phi_{1}-\Phi_{2}=I+K_{21}+\left(b_{1}-b_{2}\right)+\left(\lambda_{1} N_{1}-\lambda_{2} N_{2}\right)+\varepsilon_{L 4}
\end{gathered}
$$

In the above equation, $P_{f}$ and $\Phi_{f}$ are the pseudorange and carrier phase measurements at frequency $f(\mathrm{~m}), K_{21}$ is the sum of the satellite and receiver code instrumental delays (or called DCB in meters), $\lambda_{f}$ is the wavelength (m) and $N_{f}$ the integer ambiguity at frequency $f$ (cycle), $b_{f}$ is a frequency-dependent bias $(\mathrm{m}), \varepsilon$ is the combination of the measurements noise and the multipath distinguishing the pseudorange $\varepsilon_{P}$ from the carrier phase $\varepsilon_{L}$. More details can be seen in Sanz et al. (2013, Chapter 4.1).

Also, $I$ is defined as the ionospheric delay in the geometry-free combination as

$$
I=\left(\alpha_{2}-\alpha_{1}\right) \text { STEC }=\frac{40.28\left(f_{1}^{2}-f_{2}^{2}\right)}{f_{1}^{2} f_{2}^{2}} 10^{16} \mathrm{STEC}
$$

with $\quad \alpha_{f}=\frac{40.28}{f^{2}} 10^{16} \mathrm{~m} / \mathrm{TECU}$, where $f$ is the frequency in $\mathrm{Hz}$.

Taking $b_{I}=b_{1}-b_{2}$, the ionospheric bias, which is the main limiting factor that prevents the raw carrier phase measurements being treated as precise pseudoranges, can be expressed as

$$
B_{I}=b_{I}+\lambda_{1} N_{1}-\lambda_{2} N_{2}
$$

Therefore, the key to using carrier phase observable in STEC retrieving resides in the ability to isolate the integer ambiguities from the estimated real parameters $B_{I}$ and explicitly fix those values to integers (Collins et al. 2008). In fact, the difference of the ionospheric observables SP4 and FL4 is in the procedure to resolve the ionospheric bias in (4).

\section{Carrier phase leveled to code ionospheric observable (SP4)}

One of the methods to solve the ionospheric bias is to compute the ionospheric bias 
aligning $L_{4}$ with $P_{4}$, i.e., averaging the difference between the carrier phase and code ionospheric observable. For a continuous arc, assuming there are $\mathrm{N}$ epochs, the ambiguity term is computed as

$$
<L_{4}-P_{4}>=\sum_{i=1}^{N}\left(L_{4}-P_{4}\right) / N=B_{I}+\varepsilon_{<p>}
$$

Where $<>$ stands for the average value for a period time of carrier phase measurements that belong to the same arc.

Combining (2) and (5), we can get the carrier phase leveled to code ionospheric observable as:

$$
\mathrm{SP}_{4}=L_{4}-B_{I}=L_{4}-<L_{4}-P_{4}>=I+K_{21}+\varepsilon_{\mathrm{SP} 4}
$$

where $\varepsilon_{\mathrm{SP} 4}$ is called the leveling error, including mainly the combination of the code and carrier phase measurement noise, as well as the multipath effects (Ciraolo et al. 2007; Brunini and Azpilicueta, 2009).

Undifferenced ambiguity-fixed carrier phase ionospheric observable (FL4)

The ionospheric bias can also be solved by performing a precise geodetic data processing. As suggested by Geng et al. (2012), the narrow-lane Fractional Cycle Bias (FCB) should be determined by ambiguity-fixed network solutions. Therefore, in this work, the ionospheric bias is obtained from the undifferenced ambiguity mapped from the Double Difference (DD) ambiguities that are fixed from a network solution (Rovira-Garcia et al. 2016a). After the IAR, the fractional part of the ionospheric bias can be removed using the combination of the wide-lane and narrow-lane FCB. Therefore, the integer part of the ionospheric bias can be expressed as follow:

$$
B_{I}-b_{I}=\lambda_{1} N_{1}-\lambda_{2} N_{2}=\frac{\lambda_{1} \lambda_{2}}{\lambda_{w} \lambda_{n}}\left(\lambda_{w} N_{w}-B_{c}\right)
$$

where $N_{w}$ is the undifferenced unambiguous wide-lane ambiguity, $B_{c}$ is the undifferenced unambiguous ionospheric-free ambiguity that is reconstructed from the 
191 integer wide-lane $N_{w}$ and narrow-lane ambiguities $N_{1}, \lambda_{w}$ is the wavelength of the

192 wide-lane and $\lambda_{n}$ is the wavelength of the narrow-lane.

193 Thus, the undifferenced ambiguity-fixed carrier phase ionospheric observable 194 can be obtained as:

$$
\mathrm{FL}_{4}=L_{4}-\left(B_{I}-b_{I}\right)=L_{4}-\frac{\lambda_{1} \lambda_{2}}{\lambda_{w} \lambda_{n}}\left(\lambda_{w} N_{w}-B_{c}\right)=I+K_{21}+\varepsilon_{\mathrm{FL} 4}
$$

where $\varepsilon_{\mathrm{FL} 4}$ is the combined effect of the measurement noise, multipath and mis-modeling effects during the undifferenced ambiguity-fixed carrier phase ionospheric observable retrieval process.

In our experiment, the FL4 observable has been computed by the research group of Astronomy and Geomatics (gAGE), from the Technical University of Catalonia (UPC) using the Fast-PPP technique (Rovira-Garcia et al. 2016b). The Fast-PPP Central Processing Facility (CPF) gathers actual dual-frequency GNSS code and carrier phase measurements from 150 receivers distributed worldwide to compute the FL4, the Fast-PPP ionospheric model and the DCBs. The Fast-PPP corrections calculated for the year 2014 are freely available to the public for research and comparison purposes at the server www.gage.upc.edu/products. The strategy employed to compute the unambiguous and unbiased FL4 observable within the Fast-PPP CPF follows the procedure explained hereafter. The first step of the practical implementation is to fix the DD wide-lane ambiguity $\Delta \nabla N_{w}$ and narrow-lane ambiguity $\Delta \nabla N_{1}$, thanks to the accurate estimation of the ionospheric-free ambiguity $\Delta \nabla B_{c}$. The second step consists in introducing these integer values as constraints in the filter, which reduces the formal error of the ambiguity $B_{I}$. These computations are done within a Kalman filter that processes the geodetic data, which is unaffected by ionosphere, and a rough ionospheric model that allows a first but coarse estimation for the DCBs, in which the DCB are assumed as a constant value for $24 \mathrm{~h}$. It is worth saying that the fixing of the DD carrier phase ambiguities is not done for all the possible pairs of receivers, which would require an unaffordable time consumption. On the contrary, DD ambiguities are computed with respect to a reduced set of 
reference receiver, as shown in Figure 1.

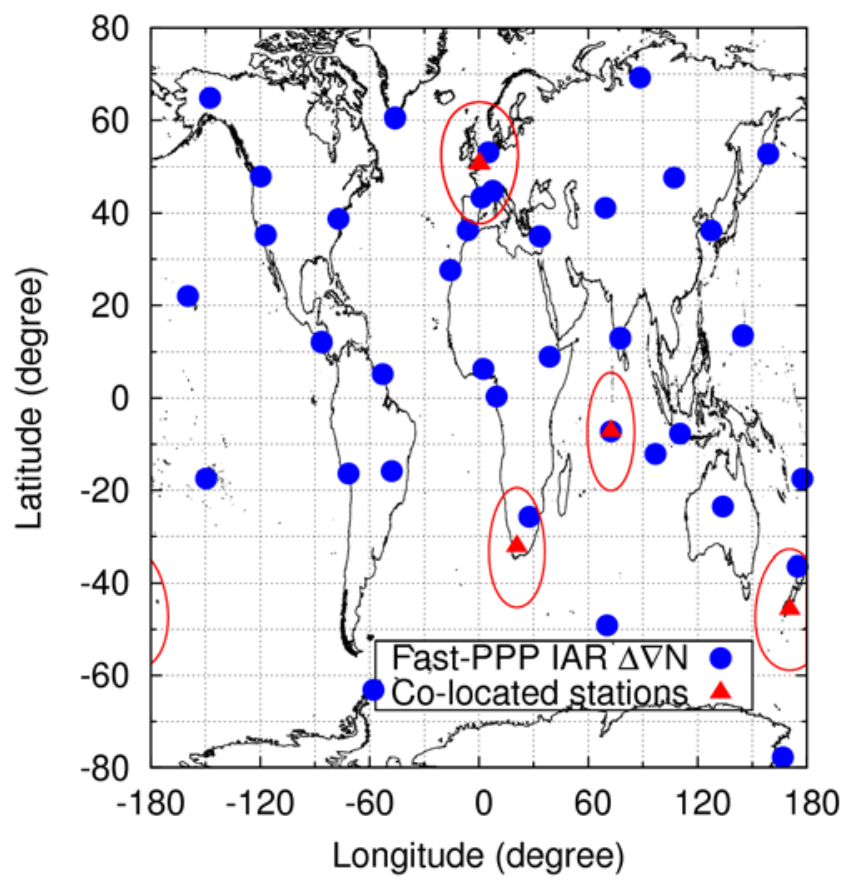

Fig. 1 Distribution of stations used in the Fast-PPP to compute the DD carrier phase ambiguities and perform IAR (blue points) together with the testing collocated station pairs (red triangles). The solid lines enclose the projection area at $268 \mathrm{~km}$ altitude, i.e., the height of the Fast-PPP first layer, for elevation angles higher than $7^{\circ}$.

A second filter runs parallel to the geodetic process; the ionospheric filter of the Fast-PPP CPF, which computes the ionospheric grid-based TEC model and refinement of the DCBs $(\triangle \mathrm{DCB})$ with respect to the coarse daily estimation into the geodetic filter. The time update in the ionospheric filter is 5 minutes for the ionospheric model parameters and 1 hour for the $\triangle \mathrm{DCB}$ refinements. The estimation is performed twice; as a forward and a mirror process. The forward process is intended for having real-time corrections, whereas the mirror process is intended for obtaining precise estimates to be used in post-process applications or in scientific studies. In this sense, in the forward process, the FL4 combinations that feed the ionospheric model in real-time, are obtained using the available estimation of the fixed ambiguity; the ambiguity is considered fixed when the formal error is below a 
The mirror process estimates the DCBs and the ionospheric model in a more robust way. In this process, each continuous arc of FL4 is obtained using the final fixed determination of the carrier phase ambiguity and the coarse DCBs estimated into the geodetic filter. Because the carrier phase ambiguities are fixed, the input data for both the forward and mirror processes should be very similar except the fact that the mirror process uses data that during the forward process were not used because the ambiguities were still not fixed. This is illustrated in Figure 2 for the receiver HERS during the day 150 in 2014. Indeed, the number of FL4s used in the mirror process, which is represented by red squares, is $34 \%$ greater than that in the forward process, which is represented by green squares. The greater number of backward measurements is especially noticeable at the beginning of the arcs.

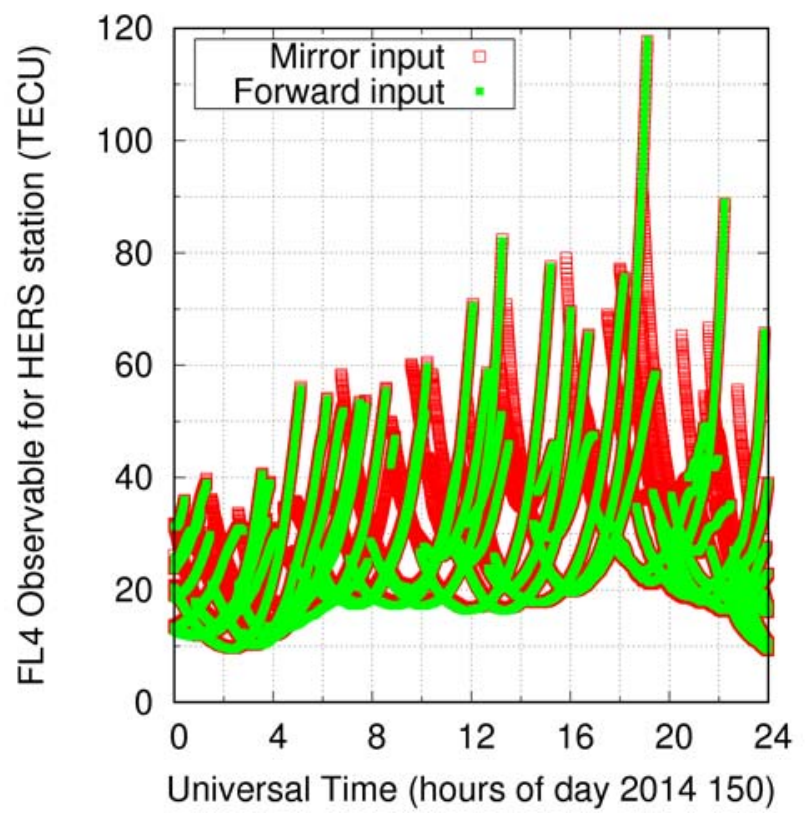

Fig. 2 Example of the FL4 measurements that feed the forward filter (in green) and the backward part of the mirror filter (in red)

To assess the effects of observation error on the ionospheric observable, the so-called 
collocated station experiment is employed (Ciraolo et al. 2007). In the set-up, permanent receivers are separated from 0 to $142 \mathrm{~m}$, so that the STEC measured by each pair of receivers is assumed to be the same. Taking Single Difference (SD) of ionospheric observables between the two receivers, for every common satellite we get a series of values free from satellite DCB, and that should ideally be the measurement noise, multipath and Between-Receiver DCB (BR-DCB).

In this manner, the previous equations, i.e., (1), (6) and (8) can be single-differenced for two collocated stations denoted " $A$ " and "B" and one observed satellite denoted as "S".

For code ionospheric observable:

$$
\Delta P_{4}=P_{4, A}^{S}-P_{4, B}^{S}=K_{21, r c v, A}-K_{21, r c v, B}+\varepsilon_{P 4, A B}
$$

For carrier phase leveled to code ionospheric observable:

$$
\Delta S P_{4}=L_{S P 4, A}^{S}-L_{S P 4, B}^{S}=K_{21, r c v, A}-K_{21, r c v, B}+\varepsilon_{S P 4, A B}
$$

For undifferenced ambiguity-fixed carrier phase ionospheric observable:

$$
\Delta F L_{4}=L_{F L 4, A}^{S}-L_{F L 4, B}^{S}=K_{21, r c v, A}-K_{21, r c v, B}+\varepsilon_{F L 4, A B}
$$

where $\Delta$ corresponds to the Single Difference operator.

According to (9) to (11), the SDs of ionospheric observable should be free from satellite DCB and that ideally be equal to the combination of the measurement noise, multipath and BR-DCB. The Standard Deviation (STD) of the time series corresponding to different satellites thus can be treated as a metric for inferring the observation error, as

where $\sqrt{2}$ stands for the SD operator under the assumption that the STD of the observation error on the collocated receivers is uncorrelated, once the ionospheric error is removed, and can be treated as the same.

The preprocessing strategy for the ionospheric observables is summarized as 
follows: (1) an elevation cut-off angle of $7^{\circ}$ is applied to filter the measurements with an elevated level of noise; (2) the carrier phase cycle-slip detection is based on

283 the geometry-free combination and the Hatch-Melbourne-Wübbena (HMW) combination (Hatch 1982; Melbourne 1985; Wübbena 1985). Specifically, to reduce the leveling errors (Ciraolo et al. 2007; Brunini and Azpilicueta, 2009) of the SP4 observable, only those arcs containing at least 120 epochs, which is 1 hour for 30-second interval data, are retained for the retrieval of the SP4 observable.

\section{Experiments and results}

The experiment has collected GPS data from nine stations, which correspond to the red triangles depicted in Figure 1. The data campaign extends the three-day period analyzed in Nie et al. (2018), comprising the Day Of Year (DOY) 150 to 180 in 2014, which is during the last solar cycle maximum. All the GPS observations have been obtained from the IGS Global Data Center at CDDIS (Crustal Dynamics Data Information System, ftp://cddis.gsfc.nasa.gov) at an interval of 30 seconds. Table 1 presents an overview of the experimental data sets ranked according to the latitude, including the four-character station name, receiver and antenna type, and the location in latitude and longitude of the receivers. According to their collocated situation, we group these receivers into four arrays with six pairs. One of the arrays contains three receivers that can be divided into three pairs, which are the SUTH-SUTM, SUTH-SUTV, and SUTM-SUTV station pairs. The other three arrays contain two receivers each that can be divided into separate pairs.

304 Table 1 An overview of the experimental data sets collected from nine IGS stations.

\begin{tabular}{lllll}
\hline Station & Receiver type & $\begin{array}{l}\text { Antenna and } \\
\text { radome type }\end{array}$ & $\begin{array}{l}\text { Location } \\
\text { Baseline } \\
\text { Length }\end{array}$ \\
\hline HERT & LEICA & LEIAT504GG & $50.7^{\circ} \mathrm{N}$ & $136 \mathrm{~m}$ \\
& GRX1200GGPRO & NONE & $0.3^{\circ} \mathrm{E}$ & \\
HERS & SEPT POLARX3ETR & LEIAR25.R3 & & \\
& & NONE &
\end{tabular}


"Revisit the calibration errors on experimental slant total electron content (TEC) determined with GPS" GPS Solutions Volume 22, Issue 85, D0I 10. 1007/s10291-018-0753-7

\begin{tabular}{lllll}
\hline DGAR & ASHTECH UZ-12 & ASH701945E_M & $7.2^{\circ} \mathrm{S}$ & $0 \mathrm{~m}$ \\
& & NONE & $72.4^{\circ} \mathrm{E}$ & \\
DGAV & JAVAD TRE_G3TH & ASH701945E_M & & \\
& & NONE & & \\
\hline SUTH & ASHTECH UZ-12 & ASH701945G_M & $32.2^{\circ} \mathrm{S}$ & $0-142 \mathrm{~m}$ \\
& & NONE & $20.8^{\circ} \mathrm{E}$ & \\
SUTV & JPS EGGDT & ASH701945G_M & & \\
& & NONE & & \\
SUTM & JAVAD TRE_G3TH & JAV_RINGANT_G3T & & \\
& & NONE & & \\
\hline OUSD & TRIMBLE NETRS & TRM55971.00 & $45.9^{\circ} \mathrm{S}$ & $3 \mathrm{~m}$ \\
& & NONE & $170.5^{\circ} \mathrm{E}$ & \\
OUS2 & JAVAD TRE_G3TH & JAV_RINGANT_G3T & & \\
& & NONE & & \\
\hline
\end{tabular}

Accuracy assessment of the ionospheric observable

The following analysis is illustrated using one collocated station pair HERT-HERS.

The results are representative of all of the other experimental pairs, summarized in what follows.

Figure 3 depicts the SD of the FL4, SP4 and RP4 observables between collocated stations HERT-HERS from DOY 150 to 180 in 2014. It is noted that the daily mean of the SD of the FL4, SP4 and RP4 observables is removed in Figure 3. From the top panel, a peak-to-peak value of about 0.15 TECU of the SD FL4 observable for the station pair HERT-HERS can be observed. With a 95\% significance, a peak-to-peak value means almost two times the STD. The middle panel depicts the SD of the SP4 observable using a y-scale ten times greater than in the top panel. It is apparent that the peak-to-peak value of the middle panel is larger than that of the top panel. The SD of the RP4 observable is presented in the bottom panel, with a peak-to-peak value of almost 10 TECU, indicating that the RP4 observable has the lowest accuracy.

In the top panel of Figure 3, the observed outliers, i.e., scatter points that deviate from the accumulation area are caused by the ionospheric bias $B_{I}$, which is still not perfectly fixed to an integer. This scattering occurs due to practical reasons in the implementation of the ambiguity-fixing strategy commented earlier. In addition, a 
324 clear daily pattern can be observed into the SD FL4 values, which will be analyzed

325 later. Apart from these, a good agreement can be found between the results in the top

326 panel from one day to another, indicating a robust ambiguity solution.

327 To quantify the reduction of the observation error, the STD of the SD FL4, SP4 328 and RP4 observable are computed every day from DOY 150 to 180 in 2014 for the 329 station pair HERS-HERT. The results are presented in Figure 4.
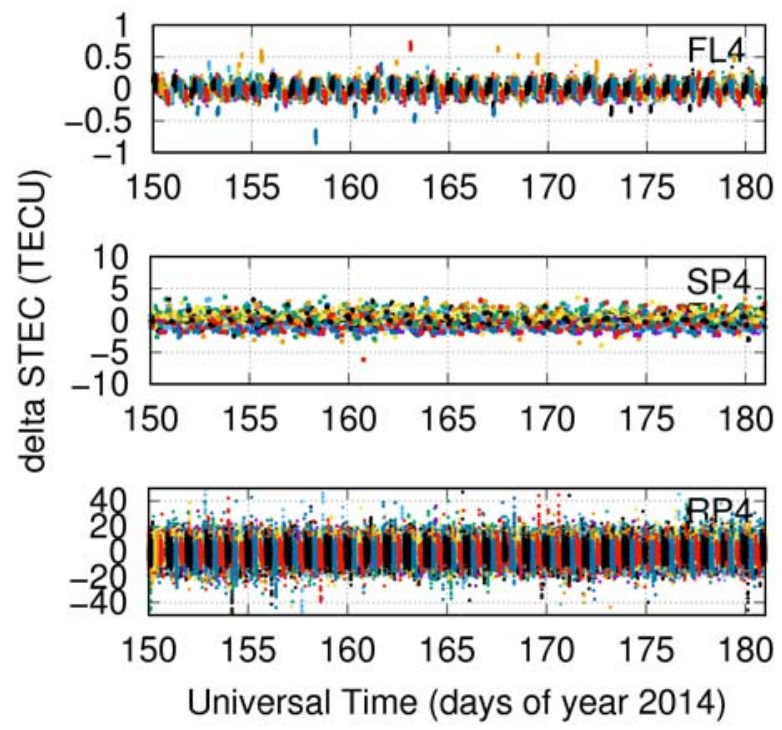

331 Fig. 3. SD ionospheric observable retrieved from collocated stations HERT-HERS

332 during DOY 150 to 180 of 2014, using FL4, SP4 and RP4 observable presented from top to bottom panel. 

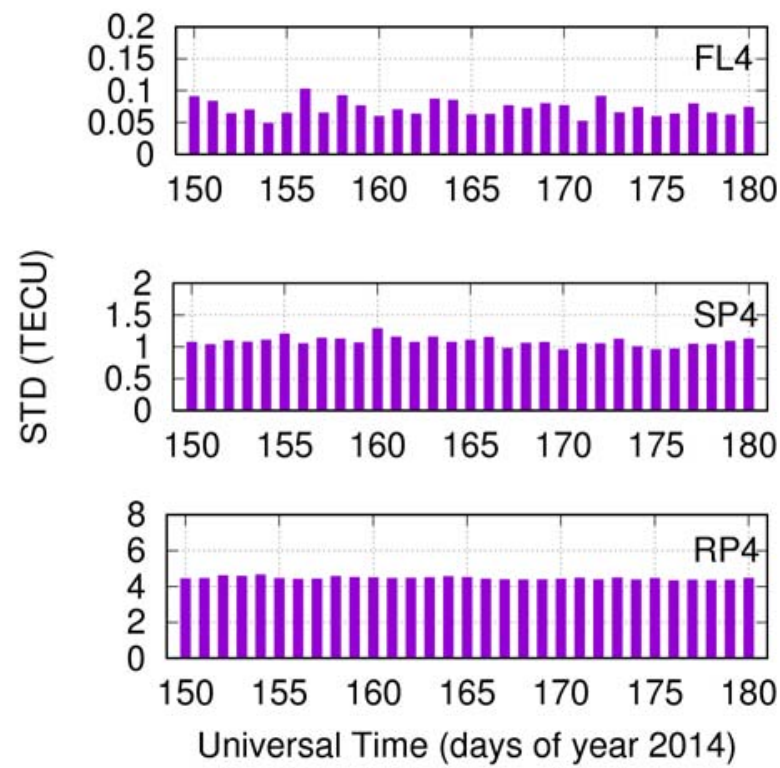

Fig. 4. Standard deviation computed every day from DOY 150 to 180 in 2014 for station pair HERS-HERT using the SD of FL4, SP4 and RP4 observable presented from top to bottom panel.

From Figure 4, we can infer that the STD of all the SD ionospheric observable during the almost one-month time, which is from DOY 150 to 180 in 2014, is quite stable. Taking the STD of FL4 observable as an example, it ranges from 0.05 to 0.10 TECU during the experiment campaign time. On the other hand, the standard deviation of the SD SP4 and RP4 observable, varying at about 1 and 4.5 TECU, respectively, can be found.

Finally, we average the STD values during the experimental time to represent the accuracy of the SD ionospheric observable. The results are presented in Table 2, which summarizes the mean, STD of the SD ionospheric observable, as well as the error of the FL4, SP4 and RP4 observable for all the six pairs of collocated stations.

Table 2 Mean, the standard deviation of the SD ionospheric observable and the Error of the FL4, SP4 and RP4 observable for all the satellites arcs for DOY 150 to 180 in 


\begin{tabular}{lcccccccccc}
\hline Baseline & \multicolumn{5}{c}{ Single difference ionospheric observable (TECU) } \\
& \multicolumn{3}{c}{ FL4 } & \multicolumn{3}{c}{ SP4 } & \multicolumn{3}{c}{ RP4 } \\
\cline { 2 - 10 } name & Mean & STD & Error & Mean & STD & Error & Mean & STD & Error \\
\hline HERT-HERS & -5.27 & 0.07 & 0.05 & -0.12 & 1.08 & 0.76 & -0.10 & 4.44 & 3.14 \\
DGAR-DGAV & 26.15 & 0.15 & 0.11 & 28.97 & 0.96 & 0.68 & 28.96 & 8.52 & 6.02 \\
SUTH-SUTM & 34.85 & 0.13 & 0.09 & 34.17 & 2.00 & 1.41 & 34.18 & 10.58 & 7.48 \\
SUTH-SUTV & 5.99 & 0.14 & 0.10 & 4.88 & 0.92 & 0.65 & 4.86 & 4.47 & 3.16 \\
SUTM-SUTV & 28.91 & 0.12 & 0.08 & 29.34 & 2.05 & 1.45 & 29.35 & 9.95 & 7.04 \\
OUS2-OUSD & 65.25 & 0.09 & 0.06 & 62.28 & 2.33 & 1.65 & 62.20 & 10.44 & 7.38 \\
\hline
\end{tabular}

Table 2 represents the performance of the FL4, SP4 and RP4 observable. It can be observed that in all baselines, the FL4 observable is more accurate, followed in order by the SP4 and RP4 observables. Numerically, the observation error affecting the FL4 observable ranges from 0.05 to 0.11 TECU whereas errors for the SP4 observable range from 0.65 to $1.65 \mathrm{TECU}$ and those for the RP4 observable range from 3.14 to 7.48 TECU. These values agree with the result presented in Ciraolo et al. (2007) and Rovira-Garcia et al. (2016a), confirming that the FL4 observable is over one order of magnitude more accurate than the SP4 and RP4 observables as was reported in the three-day study period of Nie et al. (2018). Also, the dependence of the observation error on the receiver/antenna configuration of the collocated stations can be found. For example, the observation error of the RP4 and SP4 observables for station pairs DGAV-DGAR, SUTH-SUTM, SUTM-SUTV and OUS2-OUSD are apparently larger than the for other station pairs. The reason may be attributed to that stations DGAV, SUTM and OUS2 are all equipped with JAVAD TRE_G3TH receivers, see Table 1.

Characterizing the time-varying receiver DCB to the observation error 
To quantify the contribution of time-varying receiver DCB to the observation error, the hourly receiver DCB has been first estimated by the Fast-PPP ionospheric model described earlier. Sanz et al. (2017) showed that the accuracy of the receiver DCB on a daily solution for the Fast-PPP technique, which means estimating receiver DCB as a constant in one day, was 0.57 TECU, while the satellite DCB accuracy was 0.20 TECU. This work has been extended to estimate the DCB on a sub-daily resolution, for example, one hour, in order to see the DCB stability in a shorter time interval. The intra-day stability, including the average, minimum and maximum STD values during DOY 150-180 of 2014, are presented in Table 3.

Table 3 Intra-day stability of the hourly receiver DCB estimated by the Fast-PPP from DOY $150-180$ of 2014.

\begin{tabular}{|c|c|c|c|c|}
\hline \multirow[b]{2}{*}{ Station } & \multicolumn{3}{|c|}{ Individual Receiver DCB } & \multirow{2}{*}{$\begin{array}{c}\text { BR-DCB } \\
\text { Mean } \\
\text { STD }(\text { TECU/ns })\end{array}$} \\
\hline & $\begin{array}{c}\text { Min } \\
\text { STD(TECU) }\end{array}$ & $\begin{array}{c}\text { Max } \\
\text { STD(TECU) }\end{array}$ & $\begin{array}{c}\text { Mean } \\
\text { STD(TECU/ns })\end{array}$ & \\
\hline HERT & 0.18 & 1.05 & $0.35(0.12)$ & \multirow{2}{*}{$0.09(0.03)$} \\
\hline HERS & 0.14 & 0.94 & $0.36(0.13)$ & \\
\hline DGAR & 0.14 & 3.30 & $0.92(0.32)$ & \multirow{2}{*}{$0.17(0.06)$} \\
\hline DGAV & 0.22 & 3.27 & $0.96(0.34)$ & \\
\hline SUTH & 0.18 & 0.83 & $0.39(0.14)$ & $0.10(0.03)$ \\
\hline SUTM & 0.15 & 0.88 & $0.36(0.13)$ & $0.16(0.06)$ \\
\hline SUTV & 0.15 & 0.94 & $0.40(0.14)$ & $0.11(0.04)$ \\
\hline OUS2 & 0.13 & 0.68 & $0.34(0.12)$ & \multirow{2}{*}{$0.06(0.02)$} \\
\hline OUSD & 0.15 & 0.67 & $0.34(0.12)$ & \\
\hline
\end{tabular}

Table 3 presents that the mean STD of the receiver DCB during the one-month time ranges from 0.34 to 0.96 TECU, which is much larger than the FL4 observation error that ranges from 0.05 to 0.11 TECU, as presented in Table 2. However, as it is known, the accuracy of the receiver DCB is affected by the error of the ionospheric model, which depends on the time and location of the receiver and can be transferred to the receiver DCB (Sanz et al. 2017). This transference error can be eliminated by using collocated receivers where the mis-modeling on the ionospheric model can be 
assumed to be similar, thus not affecting to the BR-DCB estimates. Therefore, the mean STD of the BR-DCB is also computed and presented in Table 3, which ranges from 0.06 to 0.17 TECU. Considering the STD of the SD FL4 observable, which ranges from 0.07 to 0.15 TECU as indicated in Table 2, we can infer that the time-varying receiver DCB contributes the most part of the observation errors of the FL4 observable that feeds the ionospheric model. This conclusion can also be seen from Figure 5, where the SD FL4 observable and BR-DCB for station pair HERT-HERS are presented together. We limit our graphical analysis to a period of four days, from DOY 150 to 153 in 2014, to present a representative result.

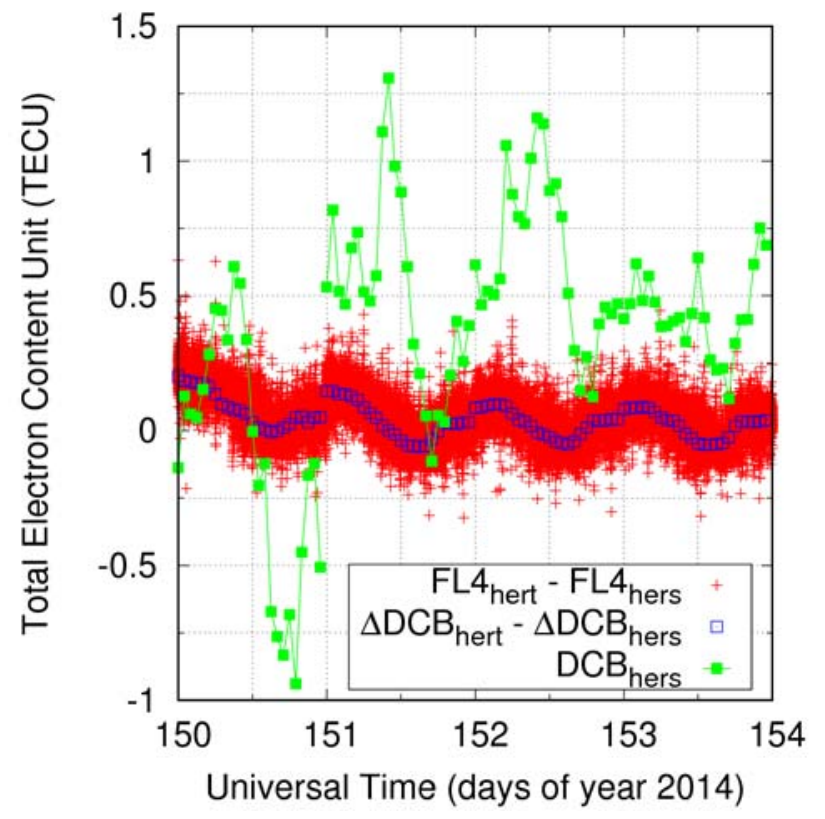

Fig. 5. SD FL4 observable, BR-DCB for station pair HERT-HERS as well as DCB of station HERS from DOY 150 to 153 in 2014

Figure 5 depicts with red pluses the SD of the FL4 observables between HERT and HERS every $30 \mathrm{~s}$, as they are input into the mirror process, which is the best determination that we can compute. In addition, the corresponding hourly BR-DCBs, which are constructed from the individual estimates from the mirror filter, are depicted with the blue rectangles. Finally, the hourly estimation of the HERS DCB, i.e. 
410 the daily rough estimation into the geodetic filter plus the refinement $\triangle \mathrm{DCB}$

411 computed by the mirror filter, is depicted with green squares. Two main

412 characteristics can be observed in Figure 5:

413 -A Day Boundary Discontinuity (DBD). These discontinuities appear because

414 carrier phase ambiguities are constrained to integer values in DD mode, whereas the

415 magnitudes depicted in Figure 5 are absolute or SD measurements. Therefore, any

416 effect that affects equally to all the satellites in view, such as a jump in the receiver

417 DCB, does not vary the value of the DD. Because carrier phase ambiguities and DCBs

418 are quite correlated, see (2), when one removes both parameters from the L4 to build

419 the FL4 measurements in (8), only small discontinuities appear in the SD

420 measurements. Thus, when common error sources, such as ionosphere, satellite orbits

421 and clock, are mitigated by building the SDs of the collocated receivers, the error of

422 the FL4, including DBD jumps, is at the level of tenths of one TECU as it can be

423 observed in Table 3 and Figure 5. Nevertheless, when undifferenced parameters are

424 considered, e.g. the DCB for the receiver HERS, the discontinuities are larger. This is

425 related with ionospheric model inaccuracies that are transferred into the parameters

426 estimation. Furthermore, due to the aforementioned correlations between carrier phase

427 ambiguities and DCBs, any error in the geodetic model is also transferred into the

428 DCBs. In any case, these discontinuities are at the level of 0.5 TECU or smaller which

429 fully agrees with the DBDs of 0.57 TECU reported in Sanz et al. (2017).

$430-$ An intra-day variability pattern. Beside the discontinuities mentioned in the 431 previous point, the absolute receiver DCB presents a clear intra-day variation that can 432 be larger than the DBDs. As it was pointed out in Sanz et al. (2017), these variations 433 can be associated to the ionospheric mis-modeling that affect the receiver DCB to a greater extent than the satellite DCBs, due to the smaller ionospheric sounding region of the stations. This mis-modeling effect is amplified in the hourly estimation of the receiver DCB compared to the daily estimate because the hourly estimation has fewer 437 observations and thus less even distribution of the observable ( $\mathrm{Li}$ et al. 2017). Moreover, the ionosphere typically repeats on a daily basis, in such a way that the 
receiver DCB estimation absorbs the mean ionospheric errors which are similar from day to day.

But, looking at the intra-day variation of the SD values, unaffected by ionospheric errors, a remaining periodic pattern can be observed in the BR-DCBs, meaning that the ionospheric model is not the only source for this intra-day variation. Using the SP4 measurements, intra-day variations of several nanoseconds in the BR-DCBs of collocated receivers have been reported by several authors (Coster et al. 2013; Zhang and Teunissen, 2015; Li et al. 2017). However, the accuracy of the FL4 observable allows detecting intra-day variations with higher accuracy. Indeed, Zhang et al. (2017) reported intra-day variations of BR-DCBs of tenths of TECU that the authors associated with temperature variations. These results agree with our results depicted in Figure 5 and in Table 3 where variations of few tenths of TECU are observed. Notice that we obtain these similar results without computing explicitly the DD of collocated receivers, as is done in Zhang et al. (2017), i.e. it is enough to do DD with respect to a reduced set of few tens of receivers distributed worldwide; see Figure 1. Finally, it is worth pointing out that these intra-day variations are observed in SD quantities, so they reflect the differential effect on the DCBs, but the effect on the undifferenced DCBs can be still larger. In other words, considering the DCBs as a constant daily parameter, as it usually assumed, can represent a mis-modeling error of at least several tenths of a TECU that can be mitigated reducing the DCB refreshing time.

\section{Effects of multipath on the observation error}

After removing the BR-DCB from the SD FL4 observable in (11), the remaining part of the observation errors includes the effects of multipath, noise and the mis-modeling of the receiver DCB. The evidence of the multipath effects, both phase multipath as well as code multipath introduced from the HMW combination, is presented in Figure 6. 


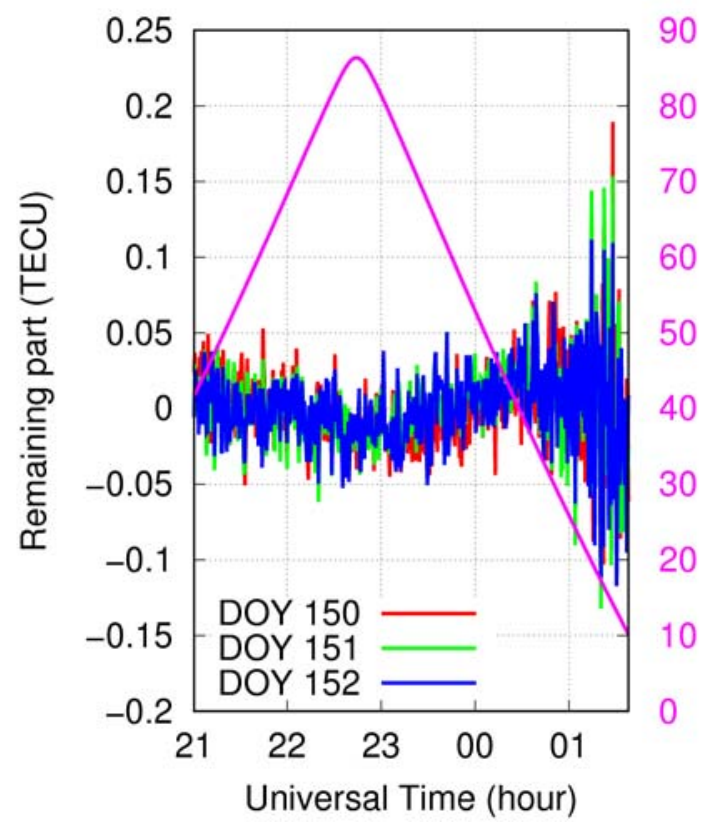

Fig. 6 Remaining part of the observation errors (with mean removed to have a better comparison) for PRN 11 by station pair HERT-HERS during DOY 150 to 152 in 2014. The results are corrected for the $3 \mathrm{~m} 56 \mathrm{~s}$ sidereal shift with respect to the first day.

In Figure 6, the remaining part of the observation errors for PRN 11 by station pair HERT-HERS during DOY 150 to 152 in 2014 are presented. As the multipath effects for the GPS constellation repeat with a sidereal day, which is $236 \mathrm{~s}$ less than a solar day, we shift UTC for the second solar day by 240 seconds and the third day by 480 seconds along the time axis to better demonstrate the sidereal day-to-day repeatability. Therefore, the multipath effects are clearly presented in the remaining part of the observation errors, especially when the elevation angle is small. Considering the magnitude of the remaining part, with a mean STD of 0.026 TECU

480 for PRN11, which is $0.026 / \sqrt{2} \times 0.162 \approx 0.003 \mathrm{~m}$ for the remaining error on GPS 481 L1 observable in the three days, the code multipath effects are much reduced. Similar conclusions can be presented by other satellites or other collocated receiver pairs. 
The objective for undertaking this investigation is to quantify the accuracy of the retrieved undifferenced ambiguity-fixed carrier phase ionospheric observable. First, the undifferenced ambiguity-fixed carrier phase ionospheric observable, FL4, is obtained through the geodetic processing to reduce the code-related multipath effect. Together with the SP4 and RP4 observable, the accuracy of the FL4 observable is evaluated through the collocated experiment. Second, because the receiver DCB varies greatly and should be carefully accounted for the ionospheric observable, its contribution to the observation errors is discussed. Also, the short-term stability of the intra-day receiver DCB has been analyzed.

(1) By using data from DOY 150-180 in 2014, the observation error affecting the undifferenced ambiguity-fixed carrier phase ionospheric observable, ranges from 0.05 to $0.11 \mathrm{TECU}$, which is over one order of magnitude less than that of the carrier phase leveled to code and the raw code ionospheric observable, which are from 0.65 to $1.65 \mathrm{TECU}$ and 3.14 to $7.48 \mathrm{TECU}$, respectively.

(2) In spite of such small error in the FL4 observables, two sources of error have been identified:

a) It has been shown that the time-varying receiver DCB, which presents clear Day Boundary Discontinuity and intra-day variability pattern, contributes the most part to the observation error. This contribution is assessed by the short-term stability of the BR-DCB, which ranges from 0.06 to 0.17 TECU in a single day.

b) Moreover, it has been shown that the remaining part of the observation errors presents a sidereal time cycle pattern, indicating that the geodetic model inaccuracies, especially the multipath, can have a small impact on the FL4 observables.

(3) Finally, by comparing the amplitude of the receiver DCB variation with respect to other error sources presented in this and in other works, we 

conclude that estimating receiver $\mathrm{DCB}$ as a daily constant can have a mis-modeling error of at least several tenths of one TECU.

\section{Acknowledgments}

The study is funded by National Key Research and Development Program of China (2016YFB0501902), National Natural Science Foundation of China (41574025, 41574013, 41731069), Spanish Ministry of Science and Innovation project (CGL2015-66410-P), The Hong Kong RGC Joint Research Scheme (E-PolyU501/16) and State Key Laboratory of Geo-Information Engineering (SKLGIE2015-M-2-2).

\section{References}

Banville S, Zhang W, Ghoddousi-Fard R, Langley, RB (2012) Ionospheric Monitoring Using Integer-Leveled Observations. Proc. ION ITM 2012, Institute of Navigation, Nashville, Tennessee, USA, September 17-21, 2692-2701.

Bossler JD, Goad CC, Bender PL (1980) Using the global positioning system (GPS) for geodetic positioning. Bulletin Géodésique 54(4):553-563.

Braasch M (1996) Multi-path effects. In: Parkinson BW Spilker JJ (eds) Global Positioning System: theory and applications, vol1. Progress in Astronautics and Aeronautics, American Institute of Aeronautics and Astronautics, 547-568

Brunini C, Azpilicueta FJ (2009) Accuracy assessment of the GPS-based slant total electron content. Journal of Geodesy 83(8):773-785.

Ciraolo L, Azpilicueta F, Brunini C, Meza A, Radicella S (2007) Calibration errors on experimental slant total electron content (TEC) determined with GPS. Journal of Geodesy 81(2):111-120

Collins P, Lahaye F, Heroux P, Bisnath S (2008) Precise point positioning with 
ambiguity resolution using the decoupled clock model. Proc. ION GNSS 2008,

Coster A, Williams J, Weatherwax A, Rideout W, Herne D (2013) Accuracy of GPS total electron content: GPS receiver bias temperature dependence. Radio Science 48(2):190-6. doi:10.1002/rds.20011.

Dow JM, Neilan RE, Rizos C (2009) The international GNSS service in a changing landscape of global navigation satellite systems. Journal of Geodesy 83(3-4):191-198.

Ge M, Gendt M, Rothacher M, Shi C, Liu J (2008) Resolution of GPS carrier phase ambiguities in precise point positioning (PPP) with daily observations. Journal of Geodesy 82(7):389-399

Geng J, Shi C, Ge M, Dodson AH, Lou Y, Zhao Q, Liu J (2012) Improving the estimation of fractional-cycle biases for ambiguity resolution in precise point positioning. Journal of Geodesy 86(8):579-89.

Hatch R (1982) The synergism of GPS code and carrier measurements. In: Proceedings of the third international symposium on satellite Doppler positioning at Physical Sciences Laboratory of New Mexico State University, Feb 8-12, 1213-1231. Schaer S, Krankowski A (2009) The IGS VTEC maps: a reliable source of ionospheric information since 1998. Journal of Geodesy 83(3-4):263-275 (2016) Comparing performances of seven different global VTEC ionospheric models in the IGS context. In International GNSS Service Workshop (IGS 2016): Sydney,

560 Australia: February 8-12, 1-13. 
positioning for GNSS Users. IEEE Transactions on Geoscience and Remote Sensing 50(10): 4213-4222.

Laurichesse D, Mercier F (2007) Integer Ambiguity resolution on undifferenced GPS phase measurements and its application to PPP. Proc. ION GNSS 2007, Institute of Navigation, , Fort Worth, Texas, USA, September 25-28, 839-848

Li M, Yuan Y, Wang N, Liu T, Chen Y (2017) Estimation and analysis of the short-term variations of multi-GNSS receiver differential code biases using global ionosphere maps. Journal of $1-15$ https://doi.org/10.1007/s00190-017-1101-3.

Liu T, Yuan Y, Zhang B, Wang N, Tang B, Cheng Y (2017) Multi-GNSS Precise Point Positioning (MGPPP) using raw observations. Journal of Geodesy 91(3):253-268

Liu T, Zhang B, Yuan Y, Li M (2018) Real-Time Precise Point Positioning (RTPPP) with raw observations and its application in real-time regional ionospheric VTEC modeling. Journal of Geodesy, 1-17. https://doi.org/10.1007/s00190-018-1118-2

Manucci AJ, Iijima BA, Lindqwister UJ, Pi X, Sparks L, Wilson BD (1999) GPS and ionosphere. URSI reviews of radio science, Jet Propulsion Laboratory, Pasadena

Melbourne WG (1985) The case for ranging in GPS-based geodetic systems. In: Proceedings of the first international symposium on precise positioning with the Global Positioning System Rockville, Maryland, April 15-19, 373-386

Nie W, Xu T, Rovira-Garcia A, Zornoza JM, Subirana JS, González-Casado G, Chen W, Xu G (2018) The Impacts of the Ionospheric Observable and Mathematical Model on the Global Ionosphere Model. Remote Sensing 10(2):169.

Rovira-Garcia A, Juan JM, Sanz J, González-Casado G (2015) A World-Wide Ionospheric Model for Fast Precise Point Positioning. IEEE Transactions on Geoscience and Remote Sensing 53(8):4596-4604. doi:10.1109/TGRS.2015.2402598 
Rovira-Garcia A, Juan JM, Sanz J, González-Casado G, Ibáñez-Segura,D (2016a)

Journal of Geodesy 90(3):229-240.doi:10.1007/s00190-015-0868-3

Rovira-Garcia A, Juan J M, Sanz J, González-Casado G, Bertran E (2016b) "Fast

Precise Point Positioning: A System to Provide Corrections for Single and

Sanz J, Juan J, Hernández-Pajares M (2013) GNSS data processing, Vol I: 978-92-9221-886-7, Noordwijk, the Netherlands

Sanz J, Juan JM, Rovira-Garcia A, González-Casado G (2017) GPS differential code biases determination: methodology and analysis. GPS Solutions 21(4):1549-1561. doi: $10.1007 / \mathrm{s} 10291-017-0634-5$

Sardon E, Rius A, Zarraoa N (1994) Estimation of the transmitter and receiver differential biases and the ionospheric total electron content from global positioning system observations. Radio Science 29(3):577-586. doi:10.1029/94RS00449

Sardon E, Zarraoa N (1997) Estimation of total electron content using GPS data: how stable are the differential satellite and receiver instrumental biases? Radio Science 32(5):1899-1910

Wilson BD, Mannucci AJ (1993) Instrumental biases in ionospheric measurement derived from GPS data. Proc. ION GPS 1993, Institute of Navigation, Salt Lake City, UT, September 22-24, 1343-1351.

Wübbena G (1985) Software developments for geodetic positioning with GPS using TI-4100 code and carrier measurements. In: Proceedings of the first international 611 symposium on precise positioning with the global positioning system, Rockville, 612 Maryland, April 15-19 
613 Zhang B, Ou J, Yuan Y, Li Z (2012) Extraction of line-of-sight ionospheric

614 observables from GPS data using precise point positioning. Science China Earth

615 Sciences 55(11):1919- 1928.

616 Zhang B, Teunissen PJ (2015) Characterization of multi-GNSS between-receiver 617 differential code biases using zero and short baselines. Science bulletin, $618 \quad 60(21): 1840-1849$.

619 Zhang B (2016). Three methods to retrieve slant total electron content measurements 620 from ground - based GPS receivers and performance assessment. Radio Science, 621 51(7):972-988.

Zhang B, Teunissen PJ, Yuan Y (2017) On the short-term temporal variations of 623 GNSS receiver differential phase biases. Journal of Geodesy 91(5):563-572. 625 positioning for the efficient and robust analysis of GPS data from large networks. 626 Journal of Geophysical Research: Solid Earth 102(B3):5005-5017. 627 doi:10.1029/96JB0

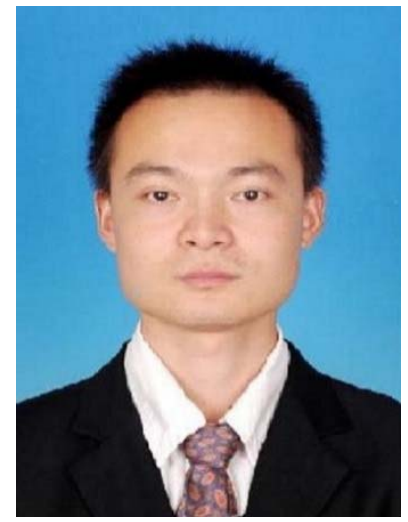

631 Wenfeng Nie is a Ph.D. candidate in the Institute of Space Sciences at Shandong 632 University, Weihai. He obtained his BSc and MSc degrees from Southeast University in 2012 and 2014, respectively. His research interests are GNSS ionospheric remote 


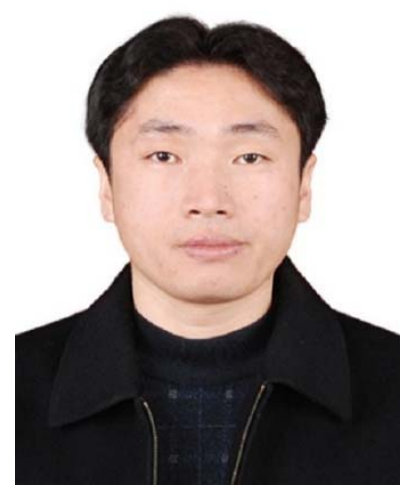

636 Tianhe $\mathbf{X u}$ is a professor in the Institute of Space Sciences at Shandong University, Weihai. He received his Ph.D. and M.S. degrees in Geodesy from the Zhengzhou Institute of Surveying and Mapping of China in 2004 and 2001. He worked in the GFZ as a visiting scientist from 2009 to 2011. His research interests are satellite navigation, orbit determination, satellite gravity data processing and quality control.

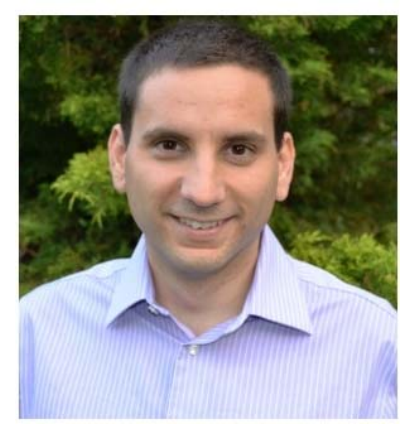

Adrià Rovira-Garcia is a post-doctoral researcher at UPC and senior research engineer of gAGE. He co-authors ten papers in peer-reviewed journals, two book chapters and over 25 works in meeting proceedings, with one best presentation award from the US Institute of Navigation and one Outstanding Poster Award from the European Geosciences Union.

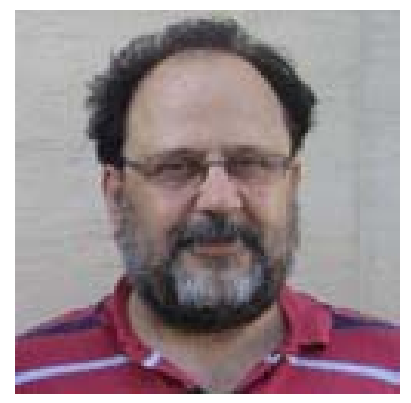


648 José Miguel Juan is with the Department of Physics (UPC) and member of gAGE

649 since 1988 . He has published over 70 papers in peer-reviewed journals and more than

650200 works in Meeting proceedings related with GNSS, with four best paper awards

651 from the US Institute of Navigation. He is a coauthor of five patents on GNSS and

652 four books on GNSS Data Processing.

653

654

655

656

657

658

659

660 Guillermo González-Casado is with the Department of Mathematics (UPC) and a

Jaume Sanz is with the Department of Mathematics (UPC) and member of gAGE since 1988. He has published over 70 papers in peer-reviewed journals and more than 200 works in meeting proceedings related with GNSS, with four best paper awards from the U.S. Institute of Navigation. He is a coauthor of five patents on GNSS and four books on GNSS Data Processing.

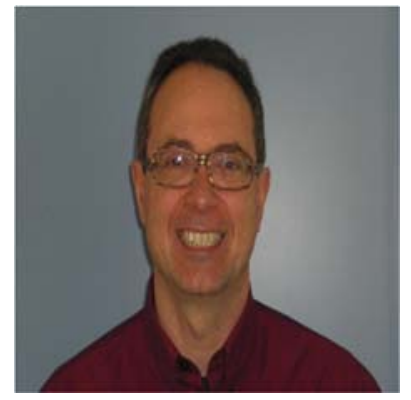
member of gAGE since 2009. His research interests are focused on ionospheric modeling based on GNSS and radio occultations, Ground and satellite-based Augmentation Systems, and the study and development of GNSS applications for the study of the ionosphere and plasmasphere.

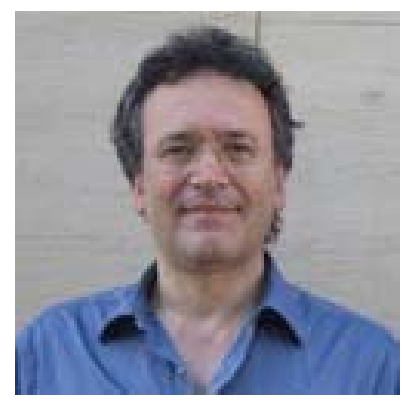


665

666

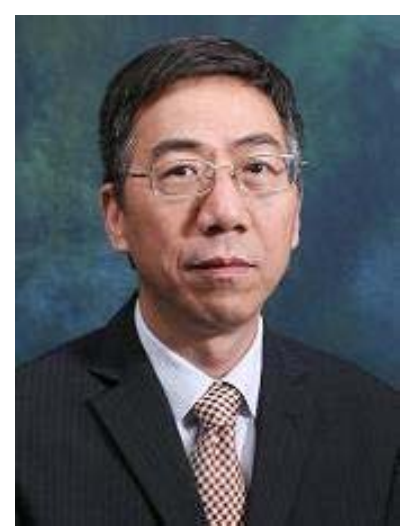

Wu Chen is a professor at the Department of Land Surveying and Geo-Informatics, Hong Kong Polytechnic University. Professor Chen has been actively working on GNSS-related research for more than 30 years. His main research interests are GNSS positioning quality evaluation, system integrity, various GNSS applications, seamless positioning and SLAM.

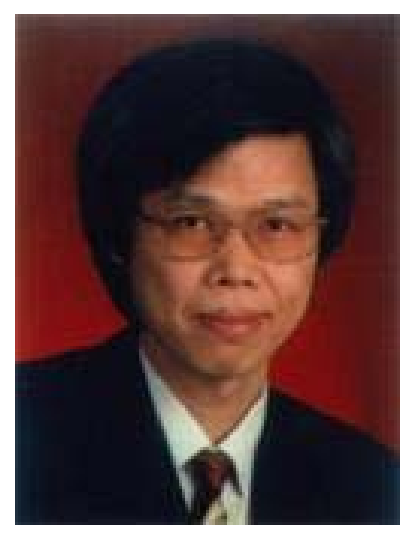

Guochang Xu obtained his Ph.D. from the Technical University Berlin in 1992. Having worked as a scientist at the GFZ from 1993 to 1998 and as a senior scientist at the National Survey and Cadastre, Denmark, from 1998 to 1999, he returned to GFZ as a senior scientist in 1999. In 2014 he was honored as the National distinguished professor by Shandong University and currently works in the Institute of Space Sciences in Shandong University at Weihai. He is the author of the books GPS (2003, 2007,2011 Chn Ed., 2014, Persian Ed.) and Orbits (2008, 2013). 